position and the number of the maxima and minima of the curve, and shows that all types actually exist. For example: for all $\delta>0$ sufficiently small, the curve

$$
\begin{aligned}
y= & (x+\delta i)(x-\delta i)\left(x+1+\delta^{3} i\right)\left(x+1-\delta^{3} i\right) . \\
& \left(x+1+\delta^{2}+\delta^{5} i\right)\left(x+1+\delta^{2}-\delta^{5} i\right)\left(x+1+\delta^{2}+\delta^{4}\right)
\end{aligned}
$$

has six extremes which, read for decreasing values of $x$, are arranged so that the first minimum of $y$ is higher than the second maximum, and the second minimum higher than the third maximum.

E. J. Moulton, Acting Secretary.

\title{
FORM OF THE NUMBER OF SUBGROUPS OF PRIME POWER GROUPS.
}

\author{
BY PROFESSOR G. A. MILLER.
}

(Read before the American Mathematical Society September 3, 1919.)

\section{\$1. Introduction.}

IT is known that the number of the subgroups of order $p^{a}, p$ being any prime number, which are contained in any group $G$ is always of the form $1+k p$. When $k=0$ for every possible pair of values for $\alpha$ and $p$ the group $G$ must be cyclic and vice versa. There are two infinite systems of groups of order $p^{m}$ containing separately $p+1$ subgroups of every order which is a proper divisor of the order of the group, viz., the abelian groups of type $(m-1,1)$ and the conformal non-abelian groups.

These two infinite systems are composed of all the groups of order $p^{m}$ involving separately exactly $p+1$ subgroups of every order which is a proper divisor of $p^{m}$. Moreover, if a group of order $p^{m}, p>2$, contains exactly $p+1$ subgroups of each of the two orders $p$ and $p^{2}$ it must contain exactly $p+1$ subgroups of every order which is a proper divisor of the order of the group, and if a group of order $2^{m}$ contains exactly three subgroups of each of the orders 2,4 and 8 it must also contain exactly three subgroups of every other order which is a proper divisor of $2^{m}$. 
The proof of the former part of this theorem may be based upon the fact that when $m>3$ such a group has to contain an invariant abelian subgroup of order $p^{3}$ and of type $(2,1)$. The order of each of the remaining operators must be divisible by $p^{3}$ and the number of the operators of order $p^{3}$ is equal to $p^{4}-p^{3}$. Hence $G$ contains exactly $p$ cyclic subgroups of order $p^{3}$ and only one non-cyclic subgroup of this order. If $m>4$, this subgroup of order $p^{4}$ must again be abelian, and by successive similar steps we note that $G$ must contain operators of order $p^{m-1}$.

When $p=2$ it is possible to extend the abelian group of order 8 and of type $(2,1)$ by 24 operators of order 4 so as to obtain a group of order 32 . In this case the preceding reasoning fails. If it is assumed, however, that $G$ contains exactly three subgroups of order 8 in addition to the three subgroups of each of the orders 2 and 4 it may be proved as in the preceding paragraph that $G$ contains only $p=2$ cyclic subgroups of order 16, etc. The theorem stated in the second paragraph has therefore been established. This theorem may be compared with the well known theorem, due to $\mathrm{W}$. Burnside, which affirms that every group of order $p^{m}, p>2$, which contains only one subgroup whose order is a proper divisor of $p^{m}$ is cyclic.

Another simple condition which implies that a group $G$ of order $p^{m}$ is cyclic is that all the operators of $G$ which transform into itself one of its subgroups constitute a cyclic subgroup of $G$. In other words, a necessary and sufficient condition that a prime power group is cyclic is that it contains at least one subgroup whose normaliser is cyclic. This theorem results directly from the fact that every non-invariant subgroup of a group of order $p^{m}$ is transformed into itself by at least $p$ of its conjugates including itself. In the following section it will be proved that a necessary and sufficient condition that an abelian group of order $p^{m}$ is cyclic is that the number of its subgroups of order $p^{a}, 0<\alpha<m$, is of the form $1+k p^{2}$ for at least one value of $\alpha$.

\section{§2. Abelian Groups.}

Let $H$ represent a subgroup of order $p^{a}$ contained in an abelian group $G$ of order $p^{m}$ and suppose that $H$ has been so selected that its invariants are as small as possible when the 
order of $H$ is fixed. It may happen that $H$ is composed of all the operators of $G$ whose orders do not exceed the largest invariant of $H$. If this is the case, $G$ contains only one subgroup of order $p^{a}$ having the same invariants as $H$. If it is not the case, we proceed to prove that the number of subgroups of $G$ which have the same invariants as $H$ must be of the form $1+p+k p^{2}$.

Let $p^{a+\lambda}$ represent the order of the subgroup of $G$ composed of all its operators whose orders divide the largest invariant of $H$. If $H$ has $\beta$ such largest invariants, the $\beta$ independent generators of $H$ whose orders are equal to these invariants can be selected from the operators of $G$ in a number of ways represented by the following product:

$$
\left(p^{a+\lambda}-p^{a-\beta}\right)\left(p^{a+\lambda}-p^{\alpha-\beta+1}\right) \cdots\left(p^{a+\lambda}-p^{a-1}\right) .
$$

Similarly, these $\beta$ independent generators can be selected from the operators of $H$ in

$$
\left(p^{a}-p^{\alpha-\beta}\right)\left(p^{\alpha}-p^{a-\beta+1}\right) \cdots\left(p^{\alpha}-p^{\alpha-1}\right)
$$

different ways, and the remaining independent generators can be selected in the same number of ways from the operators of $G$ and those of $H$. When $\lambda>0$, the quotient of the first of these two products divided by the second is evidently of the form $1+p+k p^{2}$, and hence the following theorem has been established:

Theonem. The number of the subgroups of order $p^{\alpha}$ contained in an abelian group of order $p^{m}$ and satisfying the condition that their invariants are as small as possible when $\alpha$ is given is either unity or of the form $1+p, \bmod p^{2}$.

As a special case of this theorem it may be noted that the number of subgroups of order $p^{a}$ contained in an abelian group of order $p^{m}, m>\alpha>0$, and of type $(1,1,1, \cdots)$ is always of the form $1+p, \bmod p^{2}$. In this special case $\alpha$ and $\beta$ are always equal to each other.

Suppose that $G$ contains only one subgroup $H$ of order $p^{a}$ such that its invariants are as small as possible and consider the subgroups of order $p^{a}$ which have the property that their largest invariant is $p$ times a largest invariant of $H$ and that a second invariant is equal to one of the largest remaining invariants of $H$ divided by $p$ while the rest of the invariants of such a subgroup $H^{\prime}$ are the same as those of $H$. We shall 
prove that the number of the subgroups of $G$ satisfying the conditions imposed on $H^{\prime}$ is always of the form $p+k p^{2}$, so that in this case the number of subgroups of $G$ which satisfy the conditions imposed on $H$ and $H^{\prime}$ is $1+p \bmod p^{2}$.

It will first be assumed that $H$ contains $\beta>2$ largest invariants and that the subgroup of $G$ composed of all its operators whose orders divide this largest invariant multiplied by $p$ is of order $p^{a+\lambda}$. Hence $\lambda \leqq \beta \leqq \alpha$. The number of ways in which a set of $\beta-1$ largest independent generators of $H^{\prime}$ can be selected from the operators of $G$ is expressed by the following product:

$$
\left(p^{\alpha+\lambda}-p^{\alpha}\right)\left(p^{\alpha}-p^{\alpha-\beta+1}\right) \cdots\left(p^{\alpha}-p^{\alpha-2}\right) .
$$

The number of ways in which these generators can be selected from the operators of $H^{\prime}$ is represented by the following product:

$$
\left(p^{a}-p^{\alpha-1}\right)\left(p^{a-1}-p^{a-\beta+1}\right) \cdots\left(p^{a-1}-p^{a-2}\right) .
$$

As the remaining independent generators of $H^{\prime}$ can be selected in the same number of ways from the operators of $G$ and from those of $H^{\prime}$, the quotient of the given products is equal to the number of subgroups of $G$ satisfying the conditions imposed on $H^{\prime}$. This number is evidently of the form $p+k p^{2}$, and hence the theorem in question has been proved whenever $\beta>2$.

When $\beta=2$ the largest independent generator of $H^{\prime}$ can be selected from the operators of $G$ and from those of $H^{\prime}$ in

$$
p^{\alpha+\lambda}-p^{\alpha} \text { and } p^{\alpha}-p^{\alpha-1}
$$

ways respectively while the remaining independent generators of $H^{\prime}$ can be selected in the same number of ways from each of these two groups. Hence the number of subgroups satisfying the conditions imposed on $H^{\prime}$ is $p\left(p^{\lambda-1}+p^{\lambda-2}+\cdots+1\right)$ in this case.

Finally, when $\beta=1$ and $H^{\prime}$ contains $\gamma>1$ next to the largest invariants, the $\gamma$ largest independent generators of $H^{\prime}$ can be selected from the operators of $G$ in

$$
\left(p^{a+1}-p^{a}\right)\left(p^{\delta}-p^{\delta-\gamma}\right) \cdots\left(p^{\delta}-p^{\delta-2}\right)
$$

different ways, $p^{\delta}$ being the order of the subgroup of $G$ composed of all its operators whose orders divide the second 
largest invariant of $H^{\prime}$. These independent generators can be selected from the operators of $H^{\prime}$ in

$$
\left(p^{\alpha}-p^{\alpha-1}\right)\left(p^{\delta-1}-p^{\delta-\gamma}\right) \cdots\left(p^{\delta-1}-p^{\delta-2}\right)
$$

different ways and hence the number of these subgroups is again of the form $p+k p^{2}$. This completes a proof of the following theorem, since the case $\gamma=1$ is evidently included therein:

TheoRem. If a subgroup $H$ of an abelian group $G$ of order $p^{m}$ is of order $p^{a}$ and composed of all the operators of $G$ whose orders divide a given number, then the number of the subgroups of $G$ whose largest invariant is p times the largest invariant of $H$ and whose second invariant is obtained by dividing by $p$ the largest of the remaining invariants of $H$, while its other invariants are the same as the rest of the invariants of $H$, is always of the form $p+k p^{2}$.

The preceding theorems have been established with a view to proving that the number of proper subgroups of order $p^{a}$ contained in a non-cyclic abelian group $G$ of order $p^{m}$ is always of the form $1+p \bmod p^{2}$. To complete the proof of this theorem it is only necessary to establish the fact that the number of subgroups of order $p^{\alpha}$ having a different type from those considered above must always be of the form $k p^{2}, k$ being a natural number. As a step in the proof of this theorem we note the following fundamental fact which entered the preceding considerations in a special form.

An independent generator of order $p^{\delta}$ of the subgroup $H$ of order $p^{a}$ can be selected, if these generators are selected in the descending order of magnitude and $k$ of them have already been selected, from the operators of $G$ in

$$
p^{r}-p^{s+k}
$$

different ways, where $p^{r}$ and $p^{8}$ represent the orders of the subgroups of $G$ composed of all its operators whose orders divide $p^{\delta}$ and $p^{\delta-1}$ respectively. Similarly, this independent generator can be selected from the operators of $H$ in

$$
p^{r^{\prime}}-p^{s^{\prime}+k}
$$

different ways, where $r^{\prime} \leqq r$ and $s^{\prime} \leqq s$. To prove the theorem in question it is therefore only necessary to prove that either some $s$ is at least two units larger than the corresponding 
$s^{\prime}$ or at least two $s^{\prime}$ s are each one unit larger than the corresponding $s^{\prime \prime}$ s.

From this fact it follows that the highest power of $p$ which divides the number of the subgroups of order $p^{a}$ and of type $\left(\alpha_{1}, \alpha_{2}, \cdots, \alpha_{\lambda}\right)$ may be found as follows: Determine the orders of the characteristic subgroups composed separately of all the operators whose orders divide $p^{a_{1}-1}, p^{a_{2}-1}, \cdots$, $p^{a_{\lambda}-1}$ in $G$ and in a particular subgroup $H$ of type $\left(\alpha_{1}, \alpha_{2}, \cdots, \alpha_{\lambda}\right)$ respectively. The product of the orders of these subgroups of $G$ divided by the product of the orders of the corresponding subgroups of $H$ gives a quotient which is the power of $p$ in question. For instance, if $G$ is of type $(6,6,5,4,2)$ and $H$ is of type $(6,3,2,1)$ the number of subgroups of $G$ which are of order $p^{12}$ and of type $(6,3,2,1)$ is divisible by

$$
p^{21+10+5-11-7-4}
$$

but not by any higher power of $p$.

In particular, it may be noted that the number of subgroups of order $p^{a}$ and of a given type is always divisible by $p^{2}$ whenever the number of the invariants of $G$ exceeds the number of the invariants of such a subgroup $H$ by 2 and at least one of the latter invariants exceeds $p$. If at least two of these invariants exceed $p$, the number of these subgroups is divisible by $p^{2}$ whenever $G$ has at least one more invariant than $H$ has. Hence when the number of the subgroups of the same type as $H$ has is not divisible by $p^{2}$ the number of invariants of $H$ is either the same as that of $G$ or one less than that of $G$ except when $H$ is of type $(1,1,1, \cdots)$.

Morever, when $H$ has one invariant less than $G$ and the number of subgroups having the same type as $H$ has is not divisible by $p^{2}$, it results from the preceding considerations that either no invariant of $H$ exceeds $p$, or that only one of these invariants exceeds $p$. In the latter case this invariant is $p^{2}$ unless $G$ has also only one invariant greater than $p$. Hence the following:

THEOREM: Whenever the number of subgroups of the same type as $H$ is not divisible by $p^{2}$ and the number of invariants of $H$ is less than the number of invariants of $G$ there is one and only one subgroup in $G$ having the same order as $H$ but smaller invariants than $H$ has.

It remains only to consider the case when the subgroups of order $p^{a}$ which have the same invariants as $H$ have as 
many invariants as $G$. Suppose that the invariants of $G$ arranged in descending order of magnitude are $p^{a_{1}}, p^{\alpha_{2}}, \cdots$, $p^{a_{\lambda}}$ while those of $H$ arranged similarly are $p^{a^{\prime}}, p^{a_{2}}, \cdots$, $p^{a^{\prime}}$. It is well known that $\alpha_{1} \geqq \alpha_{1}{ }^{\prime}, \alpha_{2} \geqq \alpha_{2}{ }^{\prime}, \cdots, \alpha_{\lambda} \geqq \alpha_{\lambda}{ }^{\prime}$. The number of the subgroups of type $\left(\alpha_{1}{ }^{\prime}, \alpha_{2}{ }^{\prime}, \cdots, \alpha_{\lambda}{ }^{\prime}\right)$ is evidently divisible by $p^{2}$ whenever $\alpha_{1}^{\prime}$ is at least two units larger than each of two other $\alpha^{\prime \prime}$ 's which are separately smaller than the corresponding $\alpha$ 's, and also when $\alpha_{1}{ }^{\prime}$ and $\alpha_{2}{ }^{\prime}$ are separately two units larger than some one $\alpha^{\prime}$ which is less than the corresponding $\alpha$, or $\alpha_{1}^{\prime}$ is at least three units larger than such an $\alpha^{\prime}$ provided this $\alpha^{\prime}$ is not $\alpha_{2}{ }^{\prime}$ and $\alpha_{2}=\alpha_{2}{ }^{\prime}+1$.

From the preceding paragraph it results that whenever the number of subgroups of type $\left(\alpha_{1}{ }^{\prime}, \alpha_{2}{ }^{\prime}, \cdots, \alpha_{\lambda}{ }^{\prime}\right)$ is not divisible by $p^{2}$ and an $\alpha_{\beta}{ }^{\prime}$ is less than $\alpha_{\beta}$ then there is at most one other $\alpha^{\prime}$ which is two units larger than $\alpha_{\beta}{ }^{\prime}$. If there is such an $\alpha^{\prime}$ it is $\alpha_{1}{ }^{\prime}$ and $\alpha_{\gamma}{ }^{\prime}, \beta>\gamma>1$, is equal to $\alpha_{\beta}{ }^{\prime}+1$. It was noted above that when these conditions are satisfied the number of subgroups of $G$ which are of type $\left(\alpha_{1}{ }^{\prime}, \alpha_{2}{ }^{\prime}, \cdots, \alpha_{\lambda}{ }^{\prime}\right)$ is of the form $p+k p^{2}$. This is also the case when $\alpha_{\lambda}{ }^{\prime}=\alpha_{\lambda}$, $\alpha_{\lambda}{ }^{\prime}-1=\alpha_{\lambda-1}, \ldots, \alpha_{3}{ }^{\prime}=\alpha_{3}, \alpha_{2}{ }^{\prime}=\alpha_{2}-1$, and $\alpha_{1}<\alpha-2$.

On the other hand, when none of the $\alpha^{\prime \prime}$ s is at least two units larger than the smallest $\alpha_{\beta}{ }^{\prime}$ which is less than $\alpha_{\beta}$ the number of subgroups of type $\left(\alpha_{1}{ }^{\prime}, \alpha_{2}{ }^{\prime}, \cdots, \alpha_{\lambda}{ }^{\prime}\right)$ was proved above to be of the form $1+p+k p^{2}$ provided there is at least one $\alpha^{\prime}$ which exceeds $\alpha_{\beta}{ }^{\prime}$. If there is no such $\alpha^{\prime}$ there is only one subgroup of the given type. These results establish, in particular, the following:

TheOREM. In any non-cyclic abelian group of order $p^{m}$ the number of the subgroups whose order is a given proper divisor of the order of the group is always of the form $1+p \bmod p^{2}$.

UNIVERSITY OF ILLINOIS. 